\title{
Consistency of Extended Nelson-Siegel Curve Families with the Ho-Lee and Hull and White Short Rate Models
}

\author{
Patricia Kisbye, Karem Meier \\ Facultad de Matemática, Astronomía, Física y Computación, Universidad Nacional de Córdoba, Córdoba, Argentina \\ Email: patricia.kisbye@unc.edu.ar
}

How to cite this paper: Kisbye, P. and Meier, K. (2017) Consistency of Extended Nelson-Siegel Curve Families with the Ho-Lee and Hull and White Short Rate Models. Journal of Mathematical Finance, 7, 919-933.

https://doi.org/10.4236/jmf.2017.74050

Received: August 28, 2017

Accepted: November 14, 2017

Published: November 17, 2017

Copyright ( 92017 by authors and Scientific Research Publishing Inc. This work is licensed under the Creative Commons Attribution International License (CC BY 4.0).

http://creativecommons.org/licenses/by/4.0/

\begin{abstract}
Nelson and Siegel curves are widely used to fit the observed term structure of interest rates in a particular date. By the other hand, several interest rate models have been developed such their initial forward rate curve can be adjusted to any observed data, as the Ho-Lee and the Hull and White one factor models. In this work we study the evolution of the forward curve process for each of these models assuming that the initial curve is of Nelson-Siegel type. We conclude that the forward curve process produces curves belonging to a parametric family of curves that can be seen as extended Nelson and Siegel curves. We show that the forward rate curve evolution has a linear or an exponential growth, depending on the particular short rate interest model. We applied the results to Argentinian short and forward rates obtained from the Lebac's bills yields using the Hull and White short rate model, showing a good estimation of the observed forward rate curve for near dates when the initial forward curve is adjusted with a Nelson and Siegel one.
\end{abstract}

\section{Keywords}

Nelson-Siegel Curves, Short Rate Interest Models, Consistency

\section{Introduction}

A standard procedure when dealing with concrete interest rate models is to calibrate the initial forward curve with the market observed data. That is the case of the Ho and Lee, and Hull and White models, where every curve can be perfectly fitted by adjusting the model parameters. By the other hand, some parametric curves are extendedly used to fit daily data, as is the case of the Nelson-Siegel curves [1]. 


$$
f_{N S}(\tau)=z_{1}+z_{2} \mathrm{e}^{-\lambda \tau}+z_{3} \tau \mathrm{e}^{-\lambda \tau}, \tau \geq 0
$$

with $z_{1}, z_{2}, z_{3}$ and $\lambda$ being specified parameters. So this means that it is possible to choose the Ho-Lee and Hull-White models parameters in such a way that the initial forward rate curve fits with a specific Nelson and Siegel curve. In this work we show that in this particular case, the following forward rate curves move on a manifold generated by specific parametric forward curves that can be written as a sum of a Nelson and Siegel curve and a linear or an exponential function, depending upon the short rate model. A Nelson-Siegel curve can be decomposed in three factors: $1, \mathrm{e}^{-\lambda \tau}$ and $\tau \mathrm{e}^{-\lambda \tau}$. The constant factor is related with the long term interest rate level. The exponential decay is the second factor, with an upward slope if $z_{2}>0$ or downward if $z_{2}<0$. The third factor gives a hump or o a trough, depending on $z_{3}$. Finally, $\lambda$ is called the shape parameter, and it determines the critical point of the third factor and the steepness of the hump/trough. (See [2]).

We prove that the forward rate curves produced by the Ho-Lee model and the Hull and White models when starting with a Nelson and Siegel curve is decomposed in four factors. Three of them are the same as in the Nelson and Siegel curve, and the fourth is a linear function $(\tau)$ in the Ho-Lee model or an exponential function $\left(c_{1} \mathrm{e}^{-a \tau}+c_{2} \mathrm{e}^{-2 a \tau}\right)$ in the Hull and White model, where $a$ is a model parameter. This result extends part of Bjork and Christensen (see [3]) paper results, where they proved that the above two models are inconsistent with a strictly Nelson-Siegel manifold.

In sections $\$ 3$ and $₫ 4$, we present the Ho-Lee and Hull-White models, and derive the formula for the corresponding forward curve. In each case, we choose a Nelson and Siegel curve as the initial forward curve and then prove that the following ones are extended Nelson-Siegel curves in the sense that they can be written as (1) plus a linear function or an exponential function.

In particular we also prove that each of these two short rate models is consistent with a forward curve manifold $\mathcal{G}^{\lambda}$, for each $\lambda>0$.

\subsection{Notation and Facts}

In this section we assume a probability space $(\Omega, \mathcal{F}, \mathcal{Q})$. Let $W(t), t \geq 0$ be a Wiener process, and $\left\{\mathcal{F}_{t}\right\}_{t \geq 0}$ be the filtration generated by $W(t)$. Let $\left(\Omega, \mathcal{F}, \mathcal{Q},\left\{\mathcal{F}_{t}\right\}_{t \geq 0}\right)$ denote the filtered probability space. A stochastic process $\alpha$ is called an adapted process if $\alpha(t)$ is $\mathcal{F}_{t}$-measurable for every $t \geq 0$. An Ito process with drift $\mu$ and volatility $\sigma$ is a stochastic process $X(t)$ such that

$$
X(t)=X(0)+\int_{0}^{t} \mu(s, X(s)) \mathrm{d} s+\int_{0}^{t} \sigma(s, X(s)) \mathrm{d} W(s), t \geq 0,
$$

where $\mu$ and $\sigma$ are adapted process and the second integral at the right hand side is an Ito integral [4]. Equation (2) is usually written in terms of a stochastic differential equation

$$
\mathrm{d} X(t)=\mu(t, X(t)) \mathrm{d} t+\sigma(t, X(t)) \mathrm{d} W(t)
$$


We also introduce the relationship between the Ito and the Stratonovich integral forms. If $X$ is an Ito process as in (3), then its Stratonovich integral form is as follows,

$$
\mathrm{d} X(t)=(\mu(t, X(t))+\phi(t, X(t))) \mathrm{d} t+\sigma(t, X(t)) o d W(t)
$$

where $o$ denotes the Stratonovich integration an $\phi(t, X(t))$ is a quadratic co-variance term, (see [4]). If $\sigma$ in (3) is deterministic, then $\phi(t, X(t))=0$.

We assume the existence of zero coupon bond market $\{P(t, T), 0 \leq t \leq T\}$, where $P(t, T)$ denotes the price at time $t$ of a bond with maturity $T$. We call this bond a $T$-bond. We assume that for each $t$, the curve $T \rightarrow P(t, T)$ is differentiable, with positive values and that $\{P(t, T), t \geq 0\}$ follows an Ito process for each $T \geq 0$. The forward rate curve associated to these bonds is given by

$$
f(t, T)=-\frac{\partial \ln P(t, T)}{\partial T},
$$

and the short interest rate is given by $r(t)=f(t, t)$. We shall suppose an arbitrage free model and we denote with $\mathcal{Q}$ the corresponding martingale measure. Every Ito process shall be described in terms of the $\mathcal{Q}$-measure. As in the Heath, Jarrow y Morton (HJM) [5] framework, we assume that the forward rate curve dynamics is given by a family of stochastic differential equations, which expression under $\mathcal{Q}$ is given by

$$
\mathrm{d} f(t, T)=\alpha(t, T) \mathrm{d} t+\sigma_{0}(t, T) \mathrm{d} W(t),
$$

with $\alpha$ and $\sigma_{0}$ adapted processes. The hypothesis of an arbitrage free market implies the HJM-drift condition on $\alpha$. More precisely

$$
\alpha(t, T)=\sigma_{0}(t, T) \int_{t}^{T} \sigma_{0}^{\prime}(t, s) \mathrm{d} s
$$

where the superscript in $\sigma^{\prime}$ denotes transpose in case of a vectorial process.

Given a $T$-bond, we denote $\tau=T-t$ the time up to maturity of the bond. The Brace and Musiela parametrization [6] describes the forward curve process in terms of $t$ and $\tau$ as follows,

$$
f_{r}(t, \tau)=f(t, t+\tau)
$$

and so $r(t)=f_{r}(t, 0)$. Under this parametrization, Equation (5) can be written as

$$
\mathrm{d} f_{r}(t, \tau)=\left(\frac{\partial}{\partial \tau} f_{r}(t, \tau)+\sigma(t, \tau) \int_{0}^{\tau} \sigma^{\prime}(t, s) \mathrm{d} s\right) \mathrm{d} t+\sigma(t, \tau) \mathrm{d} W(t),
$$

where $\sigma(t, \tau)=\sigma_{0}(t, t+\tau)$.

In particular, the forward rate process (8) can be expressed in terms of the Stratonovich integral form as

$$
\mathrm{d} f_{r}(t, \tau)=\left(\frac{\partial}{\partial \tau} f_{r}(t, \tau)+\sigma(t, \tau) \int_{0}^{\tau} \sigma^{\prime}(t, s) \mathrm{d} s+\phi(t, \tau)\right) \mathrm{d} t+\sigma(t, \tau) o \mathrm{~d} W(t) .
$$

\subsection{Consistency}

Consistency between short rate models and forward curves manifolds were 
stated by Bjork and Christensen in [3]. To make this work more self-contained, we recall some of their definitions and the main theorem. Let $\mathcal{M}$ a given one factor interest rate model specifying a forward rate process $f_{r}(t, \cdot)$. In terms of the Musiela parametrization, $f_{r}$ satisfies a stochastic differential equation:

$$
\mathrm{d} f_{r}(t, \tau)=\left(\frac{\partial}{\partial \tau} f_{r}(t, \tau)+\alpha(t, \tau)\right) \mathrm{d} t+\sigma(t, \tau) \mathrm{d} W(t),
$$

$t \geq 0, \tau \geq 0$, where $\alpha$ and $\sigma$ are adapted processes. In particular, the no arbitrage Heath, Jarrow and Morton (HJM) drift condition implies that $\alpha(t, x)=\sigma(t, x) \int_{t}^{x} \sigma(t, s) \mathrm{d} s$. Also, using the Stratonovich integral form, (10) can be written as

$$
\mathrm{d} f_{r}(t, \tau)=\left(\frac{\partial}{\partial \tau} f_{r}(t, \tau)+\sigma(t, x) \int_{t}^{\tau} \sigma(t, s) \mathrm{d} s+\phi(t, \tau)\right) \mathrm{d} t+\sigma(t, \tau) o \mathrm{~d} W(t),
$$

Bjork and Christensen [3] stated the following definitions of consistency between a short rate model and a parametric family of curves. At first, let $\mathcal{Z} \subseteq \mathbb{R}^{d}$ be a set of parameters, and let $G: \mathcal{Z} \rightarrow C[0, \infty)$ be a smooth function. The forward curve manifold $\mathcal{G}$ is defined as $\mathcal{G}=\operatorname{Im}(G)$. That is,

$$
\mathcal{G}=\{G(\cdot ; z):[0, \infty) \rightarrow \mathbb{R}\},
$$

where, with some abuse of notation $G(\cdot ; z)$ denotes the function $G(z)$.

Definition 1. (invariance). Consider a given interest rate model $\mathcal{M}$, specifying a forward rate process $f_{r}(t, \cdot)$, and a forward curve manifold $\mathcal{G}$. We say that $\mathcal{G}$ is invariant under the action of $f_{r}$ if, for every fixed initial time $s$, the condition $f_{r}(s, \cdot) \in \mathcal{G}$ implies that $f_{r}(t, \cdot) \in \mathcal{G}$, for all $t \geq s$, a.s.

Bjork and Christensen also stated a more restricted concept of invariance, the $f_{r}$-invariance.

Definition 2. ( $f_{r}$-invariance). Consider a given interest rate model $\mathcal{M}$, specifying a forward rate process $f_{r}(t, \cdot)$ as in (11), as well as a forward curve manifold $\mathcal{G}$. We say that $\mathcal{G}$ is $f_{r}$-invariant under the action of the forward rate process $f_{r}(t, \cdot)$ if there exists a stochastic process $Z$ with state process $\mathcal{Z}$ and possessing a Stratonovich differential of the form $\mathrm{d} Z(t)=\gamma(t, Z(t)) \mathrm{d} t+\psi(t, Z(t)) o \mathrm{~d} W(t)$, such that, for every fixed choice of initial time $s$, whenever $y(s, \cdot) \in \mathcal{G}$, the stochastic process defined by

$$
y(t, \tau)=G(\tau, Z(t)), \forall t \geq s, x \geq 0,
$$

satisfies the $\operatorname{SDE}(11)$ with initial condition $f_{r}(s, \cdot)=y(s, \cdot)$.

In this case, we say that the short rate model $\mathcal{M}$ and the manifold $\mathcal{G}$ are consistent. It is easy to prove that $f_{r}$-invariance implies invariance. Moreover, Bjork and Christensen proved the following theorem.

Theorem 3. The forward curve manifold $G$ is $f_{r}$-invariant for the forward rate process $f_{r}(t, \cdot)$ in $\mathcal{M}$ if and only if

$$
\begin{gathered}
G_{\tau}(\cdot, z)+\sigma(t, \cdot) \int_{0}^{(\cdot)} \sigma^{\prime}(t, s) \mathrm{d} s+\phi(t, \cdot) \in \operatorname{Im}\left[G_{z}(\cdot ; z)\right] \\
\sigma(t, \cdot) \in \operatorname{Im}\left[G_{z}(\cdot ; z)\right]
\end{gathered}
$$


for all $(t, z) \in[0, \infty) \times \mathcal{Z} . G_{\tau}$ and $G_{z}$ denote the Frechet derivatives of $G$ with respect to $\tau$ and $z$, which are assumed to exist.

Definition 4. An interest rate model $\mathcal{M}$ is consistent with the forward rate manifold $G$ if the consistent drift and volatility Conditions (12)-(13) hold.

\section{The Ho-Lee Short Rate Model}

The short rate model proposed by Ho and Lee [7], (henceforth HL) has a dynamic given by the $r$-process

$$
\mathrm{d} r(t)=\theta(t) \mathrm{d} t+\sigma \mathrm{d} W(t)
$$

In (14), $\{W(t), t \geq 0\}$ is a Wiener process, $\sigma>0$ and $\theta$ is a deterministic function. The HL model belongs to the family of affine short rate models. That is, if $P(t, T)$ denotes the price at time $t$ of a zero coupon bond with maturity $T$, then the term structure of the interest rate is given by:

$$
P(t, T)=\mathrm{e}^{A(t, T)-r(t) B(t, T)},
$$

for certain functions $A$ and $B$. In particular, in the case of the Ho-Lee model, $A$ and $B$ are given by:

$$
\begin{gathered}
B(t, T)=T-t \\
A(t, T)=\int_{t}^{T} \theta(s)(s-T) \mathrm{d} s+\frac{\sigma^{2}(T-t)^{3}}{6}
\end{gathered}
$$

(see for instance [8]). The forward rate curve is related with the term structure by the equation:

$$
f(t, T)=-\frac{\partial \ln (P(t, T))}{\partial T}=-\frac{\partial A(t, T)}{\partial T}+\frac{\partial B(t, T) r(t)}{\partial T}
$$

Then, in this case replacing $A$ and $B$ by the expressions in (17) and (16), we get:

$$
\begin{aligned}
f(t, T) & =\frac{\partial}{\partial T}\left(-\int_{t}^{T} \theta(s)(s-T) \mathrm{d} s-\frac{\sigma^{2}(T-t)^{3}}{6}\right)+r(t) \frac{\partial}{\partial T}(T-t) \\
& =-\left(\theta(T) T-\int_{t}^{T} \theta(s) \mathrm{d} s-T \theta(T)\right)-\frac{\sigma^{2}}{2}(T-t)^{2}+r(t) \\
& =\int_{t}^{T} \theta(s) \mathrm{d} s-\frac{\sigma^{2}}{2}(T-t)^{2}+r(t)
\end{aligned}
$$

In particular it holds that $f(t, t)=r(t)$. If $T \rightarrow f^{*}(0, T)$ is the observed initial forward curve, and $\theta$ is defined as

$$
\theta(t)=\sigma^{2} t+\frac{\partial f^{*}}{\partial T}(0, t)
$$

then $f(0, T)=f^{*}(0, T)$. That is, the model parameters can be adjusted such that the initial forward curve fits the observed one.

We now assume that the initial forward curve is given by a Nelson and Siegel parametric curve. That is, we define: 


$$
f^{*}(0, T)=z_{1}+z_{2} \mathrm{e}^{-\lambda T}+z_{3} T \mathrm{e}^{-\lambda T}, T \geq 0
$$

where $z_{1}, z_{2}, z_{3}$ and $\lambda$ are fixed real numbers, $\lambda>0$. We want to study the evolution of this initial curve in the $t$ variable. With this particular choice of $f^{*}, \theta$ is given by:

$$
\theta(t)=\sigma^{2} t+\left(z_{3}-z_{2} \lambda\right) \mathrm{e}^{-\lambda t}-z_{3} \lambda t \mathrm{e}^{-\lambda t}
$$

and the solution of the Ho-Lee stochastic differential Equation (14) is given by:

$$
\begin{aligned}
r(t) & =r(0)+\frac{\sigma^{2} t^{2}}{2}-\frac{z_{3}-z_{2} \lambda}{\lambda} \mathrm{e}^{-\lambda t}-\frac{z_{3}}{\lambda}\left(1-\mathrm{e}^{-\lambda t}(\lambda t+1)\right)+\sigma W(t) \\
& =r(0)+\frac{\sigma^{2} t^{2}}{2}-\frac{z_{3}}{\lambda}+\left(z_{2}+z_{3} t\right) \mathrm{e}^{-\lambda t}+\sigma W(t)
\end{aligned}
$$

where $r(0)$ is the short rate value at time $t=0$.

We compute the integral term in (19):

$$
\begin{aligned}
& \int_{t}^{T} \theta(s) \mathrm{d} s=\int_{t}^{T} \sigma^{2} s+\left(z_{3}-z_{2} \lambda\right) \mathrm{e}^{-\lambda s}-z_{3} \lambda t \mathrm{e}^{-\lambda s} \mathrm{~d} s \\
& =\frac{\sigma^{2}}{2}\left(T^{2}-t^{2}\right)+\left(z_{2}+z_{3} T\right) \mathrm{e}^{-\lambda T}-\left(z_{2}+z_{3} t\right) \mathrm{e}^{-\lambda t}
\end{aligned}
$$

We can now derive an explicit formula for the forward rate curve process:

$$
\begin{aligned}
f(t, T) & =\frac{\sigma^{2}}{2}\left(T^{2}-t^{2}\right)+\left(z_{2}+z_{3} T\right) \mathrm{e}^{-\lambda T}-\left(z_{2}+z_{3} t\right) \mathrm{e}^{-\lambda t}-\frac{\sigma^{2}}{2}(T-t)^{2}+r(t) \\
& =\sigma^{2} t(T-t)+\left(z_{2}+z_{3} T\right) \mathrm{e}^{-\lambda T}-\left(z_{2}+z_{3} t\right) \mathrm{e}^{-\lambda t}+r(t)
\end{aligned}
$$

The above computations allow us to state the following theorem.

Theorem 5. Let $r$ denote the HL short rate with a dynamic as stated in (14). Then, if the initial forward rate curve is a Nelson and Siegel parametric curve as in (20), the corresponding forward rate curve at time $t$ is given by the formula

$$
f(t, T)=r(t)+\sigma^{2} t(T-t)+\left(z_{2}+z_{3} T\right) \mathrm{e}^{-\lambda T}-\left(z_{2}+z_{3} t\right) \mathrm{e}^{-\lambda t}
$$

Let $\tau=T-t$ the time up to maturity. Using the Brace and Musiela parametrization, we denote $f_{H L}$ the forward curve given by $f_{H L}(t, \tau)=f(t, t+\tau)$. Then the forward rate curve $f_{H L}$ has the expression

$$
f_{H L}(t, \tau)=\sigma^{2} t \tau+C_{1}(t)+C_{2}(t) \mathrm{e}^{-\lambda \tau}+C_{3}(t) \tau \mathrm{e}^{-\lambda \tau}, t \geq 0, \tau \geq 0,
$$

where $C_{1}, C_{2}, C_{3}$ are coefficients that depends on $\mathrm{t}$ and the Nelson and Siegel parameters:

$$
\begin{gathered}
C_{1}(t)=r(t)-\left(z_{2}+z_{3} t\right) \mathrm{e}^{-\lambda t} \\
C_{2}(t)=\left(z_{2}+z_{3} t\right) \mathrm{e}^{-\lambda t} \\
C_{3}(t)=z_{3} \mathrm{e}^{-\lambda t}
\end{gathered}
$$

Proof. The proof follows arranging terms after replacing $T$ by $\tau+t$ in Equation (21).

The expression of the function $\tau \rightarrow f_{H L}(t, \tau)$ given in the formula (22) is a sum of a linear function plus a Nelson and Siegel parametric curve. 
Definition 6. Let $\lambda>0$ and $g:[0, \infty) \rightarrow \mathbb{R}$ be a function defined as

$$
g(\tau)=z_{0} \tau+z_{1}+z_{2} \mathrm{e}^{-\lambda t}+z_{3} \mathrm{e}^{-\lambda t},
$$

where $z_{0}, z_{1}$ and $z_{3}$ are constant real numbers. We call $g$ a linearly extended Nelson-Siegel curve.

In particular, in the following subsection we study the consistency of the Ho-Lee model with a family of forward curve manifolds $\mathcal{G}^{\lambda}$ generated by linearly extended Nelson-Siegel curves.

\section{Consistency between the HL Model and Forward Curve Manifolds}

The forward rate curve $f$ given in (21) satisfies the Heath, Jarrow and Morton drift condition:

$$
\alpha(t, T)=\sigma(t, T) \int_{t}^{T} \sigma(t, s) \mathrm{d} s=\sigma^{2}(T-t),
$$

and the stochastic differential equation

$$
\mathrm{d} f(t, T)=\alpha(t, T) \mathrm{d} t+\sigma(t, T) \mathrm{d} W(t)=\sigma^{2}(T-t) \mathrm{d} t+\sigma \mathrm{d} W(t),
$$

with $T \geq 0$ and $0 \leq t \leq T$. In terms of the Brace and Musiela parametrization, fHL satisfies:

$$
\mathrm{d} f_{H L}(t, \tau)=\left(\frac{\partial}{\partial \tau} f_{H L}(t, \tau)+\sigma^{2} \tau\right) \mathrm{d} t+\sigma \mathrm{d} W(t),
$$

$t \geq 0, \tau \geq 0$. Starting from Equations (22) and (23), our conjecture is that there exists a forward curve manifold containing linearly extended Nelson-Siegel curves that is consistent with the HL model. In fact, this is stated and proved by the following theorem.

Theorem 7. Let $\mathcal{Z}=\mathbb{R}^{4}, \lambda>0$ and

$$
G^{\lambda}(\tau ; \beta)=\beta_{0} \tau+\beta_{1}+\beta_{2} \mathrm{e}^{-\lambda \tau}+\beta_{3} \tau \mathrm{e}^{-\lambda \tau}, \tau \geq 0
$$

Let $f_{H L}(t,$.$) be the Ho-Lee forward rate process. Then, for each \lambda>0$ the forward curve manifold $\mathcal{G}^{\lambda}$ is $f_{H L}$-invariant.

Proof. We shall apply Theorem 3 to see that $\mathcal{G}^{\lambda}$ is $f_{H L}$-invariant. Because the volatility term in (23) is deterministic, the standard differential equation is the same for the Ito and the Stratonovich integral formulation. The Frechet derivatives of $G^{\lambda}$ are given by:

$$
\begin{gathered}
G_{\beta}^{\lambda}(\tau, \beta)=\left[\tau, 1, \mathrm{e}^{-\lambda \tau}, \tau, \mathrm{e}^{-\lambda \tau}\right] \\
G_{\tau}^{\lambda}(\tau, \beta)=\beta_{0}+\left(-\beta_{2} \lambda+\beta_{3}\right) \mathrm{e}^{-\lambda \tau}-\beta_{3} \lambda \tau \mathrm{e}^{-\lambda \tau}
\end{gathered}
$$

In order to prove that $\mathcal{G}^{\lambda}$ is $f_{H L}$-invariant, we must check the drift and volatility consistency Conditions (12) and (13). We shall first prove that $G_{\tau}^{\lambda}(\cdot, \beta)+\sigma^{2}(\cdot) \in \operatorname{Im}\left[G_{\beta}^{\lambda}(\cdot, \beta)\right]$. This means that there must be real numbers $A, B, C$ and $D$ such that:

$$
\beta_{0}+\left(-\beta_{2} \lambda+\beta_{3}\right) \mathrm{e}^{-\lambda \tau}-\beta_{3} \lambda \tau \mathrm{e}^{-\lambda \tau}+\sigma^{2} \tau=A \tau+B+C \mathrm{e}^{-\lambda \tau}+D \tau \mathrm{e}^{-\lambda \tau}
$$


In fact, this is possible taking

$$
A=\sigma^{2}, B=\beta_{0}, C=-\beta_{2} \lambda+\beta_{3} \text { and } D=-\beta_{3} \lambda
$$

so Condition (12) is satisfied. To prove Condition (13), we must find $A, B, C$ and $D$ such that

$$
\sigma=A \tau+B+C \mathrm{e}^{-\lambda \tau}+D \tau \mathrm{e}^{-\lambda \tau}
$$

and this can be done taking $A=B=D=0$ and $B=\sigma$.

Theorem 7 implies that the forward curve manifold $G^{\lambda}$ is $f_{H L}$-invariant, so the next corollary follows:

Corollary 1 . For every $\lambda>0$, the forward curve manifold $G^{\lambda}$ is consistent with the Ho-Lee short rate model.

Corollary 1 implies that, in the particular case that $\theta$ in (14) is chosen such that the initial forward rate curve fits the Nelson and Siegel curve

$$
f^{*}(0, \tau)=z_{1}+z_{2} \mathrm{e}^{-\lambda \tau}+z_{3} \tau \mathrm{e}^{-\lambda \tau}, \tau \geq 0
$$

then, for each $t \geq 0$ the corresponding forward rate curve $f_{H L}(t, \tau)$ can be written as a linearly extended Nelson and Siegel curve. That is:

$$
f_{H L}(t, \tau)=\beta_{0} \tau+\beta_{1}+\beta_{2} \mathrm{e}^{-\beta_{4} \tau}+\beta_{3} \tau \mathrm{e}^{-\beta_{4} \tau}, \tau \geq 0
$$

then the drift consistency condition is not satisfied, because it requires $\beta_{4}=\lambda$.

\section{The Hull and White Model}

The short rate model proposed by Hull and White [9], (henceforth HW) or extended Vasicek model has the following stochastic differential equation:

$$
\mathrm{d} r(t)=(\theta(t)-\operatorname{ar}(t)) \mathrm{d} t+\sigma \mathrm{d} W(t)
$$

where $a, \sigma$ are positive real numbers and $\theta$ is a deterministic function. $\theta$ can be chosen in such a way that the initial forward curve $f(0, \cdot)$ fits with the observed data at $t=0$. Let $f^{*}(0, \cdot)$ be a particular forward curve observed at $t=0$. Then $\theta$ is defined as:

$$
\theta(t)=\frac{\partial f^{*}}{\partial T}(0, t)+a f^{*}(0, t)+\frac{\sigma^{2}}{2 a}\left(1-\mathrm{e}^{-2 a t}\right)
$$

The Hull and White short rate model belongs also to the class of affine models, and in this case the functions $A$ and $B$ in Equation (15) are given by [8]:

$$
\begin{gathered}
B(t, T)=\frac{1}{a}\left(1-\mathrm{e}^{-a(T-t)}\right) \\
A(t, T)=\int_{t}^{T}\left(\frac{1}{2} \sigma^{2} B^{2}(s, T)-\theta(s) B(s, T)\right) \mathrm{d} s
\end{gathered}
$$

The corresponding forward rate curve is given by:

$$
\begin{aligned}
f(t, T) & =-\frac{\partial A(t, T)}{\partial T}+\frac{\partial B(t, T) r(t)}{\partial T} \\
& =-\frac{\partial}{\partial T}\left(\int_{t}^{T}\left(\frac{1}{2} \sigma^{2} B^{2}(s, T)-\theta(s) B(s, T)\right) \mathrm{d} s\right)+r(t) \mathrm{e}^{-a(T-t)}
\end{aligned}
$$


Now, by Leibniz rule, we have:

$$
\begin{aligned}
\frac{\partial}{\partial T}\left(\int_{t}^{T} \frac{1}{2} \sigma^{2} B^{2}(s, T) \mathrm{d} s\right) & =\frac{1}{2} \sigma^{2} B^{2}(T, T)+\int_{t}^{T} \frac{1}{2} \sigma^{2} \frac{\partial B^{2}(s, T)}{\partial T} \mathrm{~d} s \\
& =\sigma^{2} \int_{t}^{T} B(s, T) \frac{\partial B(s, T)}{\partial T} \mathrm{~d} s \\
& =\sigma^{2} \int_{t}^{T} \frac{1-\mathrm{e}^{-a(T-s)}}{a} \mathrm{e}^{-a(T-s)} \mathrm{d} s \\
& =-\frac{1}{2} \sigma^{2} B^{2}(t, T)=-\frac{\sigma^{2}}{2 a^{2}}\left(1-\mathrm{e}^{-a(T-t)}\right)^{2} \\
\frac{\partial}{\partial T}\left(\int_{t}^{T} \theta(s) B(s, T)\right) & =\theta(T) B(T, T)+\int_{t}^{T} \theta(s) \frac{\partial B(s, T)}{\partial T} \mathrm{~d} s \\
& =\int_{t}^{T} \theta(s) \mathrm{e}^{-a(T-s)} \mathrm{d} s
\end{aligned}
$$

We now assume that the initial forward curve is fitted to a Nelson and Siegel parametric curve,

$$
f^{*}(0, T)=z_{1}+z_{2} \mathrm{e}^{-\lambda T}+z_{3} T \mathrm{e}^{-\lambda T}, T \geq 0
$$

Then, the solution of (24) and the function $\theta$ are given by the following expressions:

$$
\begin{gathered}
r(t)=r(0) \mathrm{e}^{-a t}+\int_{0}^{t} \mathrm{e}^{-a(t-u)} \theta(u) \mathrm{d} u+\sigma \int_{0}^{t} \mathrm{e}^{-a(t-u)} \mathrm{d} W(u) \\
=r(0) \mathrm{e}^{-a t}+\alpha(t)-\alpha(0) \mathrm{e}^{-a t}+\sigma \int_{0}^{t} \mathrm{e}^{-a(t-u)} \mathrm{d} W(u) \\
\theta(t)=a z_{1}+\left(z_{3}-z_{2} \lambda+a z_{2}\right) \mathrm{e}^{-\lambda t}+\left(a z_{3}-z_{3} \lambda\right) t \mathrm{e}^{-\lambda t}+\frac{\sigma^{2}}{2 a}\left(1-\mathrm{e}^{-2 a t}\right)
\end{gathered}
$$

where

$$
\alpha(t)=f^{*}(0, t)+\frac{\sigma^{2}}{2 a^{2}}\left(1-\mathrm{e}^{-a t}\right)^{2} .
$$

Now we can compute explicitly

$$
\begin{aligned}
\int_{t}^{T} \theta(s) \mathrm{e}^{-a(T-s)} \mathrm{d} s & =\alpha(T)-\alpha(t) \mathrm{e}^{-a(T-t)} \\
& =f^{*}(0, T)+\frac{\sigma^{2}}{2 a^{2}}\left(1-\mathrm{e}^{-a T}\right)^{2}-\alpha(t) \mathrm{e}^{-a(T-t)}
\end{aligned}
$$

Replacing the Expressions (29) and (30) in the forward rate curve formula (28), we get:

$f(t, T)=-\frac{\sigma^{2}}{2 a^{2}}\left(1-\mathrm{e}^{-a(T-t)}\right)^{2}+f^{*}(0, T)+\frac{\sigma^{2}}{2 a^{2}}\left(1-\mathrm{e}^{-a T}\right)^{2}-\alpha(t) \mathrm{e}^{-a(T-t)}+r(t) \mathrm{e}^{-a(T-t)}$

With the above computations we arrive to the next theorem.

Theorem 8. Let $r$ denote the Hull and White short rate model with the dynamics stated in (24). Then, if the initial forward rate curve is a Nelson and Siegel parametric curve as in (20), the corresponding forward rate curve at time $t$ is given by the formula

$f(t, T)=-\frac{\sigma^{2}}{2 a^{2}}\left(1-\mathrm{e}^{-a(T-t)}\right)^{2}+f^{*}(0, T)+\frac{\sigma^{2}}{2 a^{2}}\left(1-\mathrm{e}^{-a T}\right)^{2}-\alpha(t) \mathrm{e}^{-a(T-t)}+r(t) \mathrm{e}^{-a(T-t)}$ 
Let $\tau=T-t$ the time up to maturity. Using the Brace and Musiela parametrization, we denote $f_{H W}$ the forward curve given by $f_{H W}(t, \tau)=f(t, t+\tau)$. Then the forward rate curve $f_{H W}$ has the expression

$$
f_{H W}(t, \tau)=C_{1}(t) \mathrm{e}^{-a \tau}+C_{2}(t) \mathrm{e}^{-2 a \tau}+C_{3}(t)+C_{4}(t) \mathrm{e}^{-\lambda \tau}+C_{5}(t) \tau \mathrm{e}^{-\lambda \tau}
$$

where $C_{1}, C_{2}, C_{3}, C_{4}$ and $C_{5}$ are coefficients that depends on $t, r(t)$ and the Nelson and Siegel curve parameters:

$$
\begin{gathered}
C_{1}(t)=\frac{\sigma^{2}}{a^{2}}\left(1-\mathrm{e}^{-a t}\right)-\alpha(t)+r(t), C_{2}(t)=\frac{\sigma^{2}}{2 a^{2}}\left(\mathrm{e}^{-2 a t}-1\right), \\
C_{3}(t)=z_{1}, C_{4}(t)=z_{2} \mathrm{e}^{-\lambda t}+z_{3} t \mathrm{e}^{-\lambda t}, C_{5}(t)=z_{3} \mathrm{e}^{-\lambda t} .
\end{gathered}
$$

The expression of the function $\tau \rightarrow f_{H W}(t, \tau)$ given in the formula (31) is a sum of an exponential function plus a Nelson and Siegel parametric curve.

Definition 9. Let $\lambda>0$ and $g:[0, \infty) \rightarrow \mathbb{R}$ be a function defined as

$$
g(\tau)=c_{1} \mathrm{e}^{-a \tau}+c_{2} \mathrm{e}^{-2 a \tau}+z_{0} \tau+z_{1}+z_{2} \mathrm{e}^{-\lambda \tau}+z_{3} \mathrm{e}^{-\lambda \tau}
$$

with $c_{1}, c_{2}, z_{0}, z_{1}$ and $z_{2}$ constant real numbers. We call $g$ an exponentially extended Nelson-Siegel curve.

In particular, in the following subsection we study the consistency of the Hull and White model with a family of forward curve manifolds $\mathcal{G}^{\lambda}$ generated by exponentially extended Nelson-Siegel curves.

\section{Consistency between the Hull and White Model and Forward Curve Manifolds}

The dynamics of the forward curve process is given in terms of the Ito integral formulation by

$$
\mathrm{d} f(t, T)=\frac{\sigma^{2}}{a} \mathrm{e}^{-a(T-t)}\left(1-\mathrm{e}^{-a(T-t)}\right) \mathrm{d} t+\sigma \mathrm{e}^{-a(T-t)} \mathrm{d} W(t),
$$

$0 \leq t<T<\infty$, and in the Musiela parametrization $\tau=T-t$,

$$
\mathrm{d} f_{H W}(t, \tau)=\left(\frac{\partial}{\partial \tau} f_{H W}(t, \tau)+\frac{\sigma^{2}}{a} \mathrm{e}^{-a \tau}\left(1-\mathrm{e}^{-a \tau}\right)\right) \mathrm{d} t+\sigma \mathrm{e}^{-a \tau} \mathrm{d} W(t)
$$

$\tau \geq 0, t \geq 0$. Because the volatility term is a deterministic function, the Stratonovich and the Ito integral formulation are the same.

We state the next theorem:

Theorem 10. Let $\mathcal{Z}=\mathbb{R}^{5}$ and for each $\lambda>0$ let $G^{\lambda}: \mathcal{Z} \rightarrow C(0, \infty)$ be defined as

$$
G^{\lambda}(\tau ; \beta)=\beta_{1} \mathrm{e}^{-a \tau}+\beta_{2} \mathrm{e}^{-2 a \tau}+\beta_{3}+\beta_{4} \mathrm{e}^{-\lambda \tau}+\beta_{5} \tau \mathrm{e}^{-\lambda \tau} .
$$

Let $f_{H W}(t, \cdot)$ be the Hull and White forward rate process given by the standard differential Equation (32). Then, the forward curve manifold $\mathcal{G}^{\lambda}$ is $f_{H W}$ invariant.

Proof. We note that the Frechet derivatives $G_{\beta}^{\lambda}$ and $G_{\tau}^{\lambda}$ are given by:

$$
G_{\beta}^{\lambda}(\tau ; \beta)=\left[\mathrm{e}^{-a \tau}, \mathrm{e}^{-2 a \tau}, 1, \mathrm{e}^{-\lambda \tau}, \tau \mathrm{e}^{-\lambda \tau}\right]
$$




$$
G_{\tau}^{\lambda}(\tau ; \beta)=\beta_{1}(-a) \mathrm{e}^{-a \tau}+\beta_{2}(-2 a) \mathrm{e}^{-2 a \tau}+\left(-\beta_{4} \lambda+\beta_{5}\right) \mathrm{e}^{-\lambda \tau}-\beta_{5} \lambda \tau \mathrm{e}^{-\lambda \tau} .
$$

So, first we prove that $G_{\tau}^{\lambda}(\because \beta)+\frac{\sigma^{2}}{a} \mathrm{e}^{-a(\cdot)}\left(1-\mathrm{e}^{-a(\cdot)}\right) \in \operatorname{Im}\left[G_{\beta}^{\lambda}(\cdot ; \beta)\right]$. So we look for real numbers $A, B, C, D$ and $E$ such that

$$
\begin{aligned}
& \beta_{1}(-a) \mathrm{e}^{-a \tau}+\beta_{2}(-2 a) \mathrm{e}^{-2 a \tau}+\left(-\beta_{4} \lambda+\beta_{5}\right) \mathrm{e}^{-\lambda \tau}-\beta_{5} \lambda \tau \mathrm{e}^{-\lambda \tau}+\frac{\sigma^{2}}{a} \mathrm{e}^{-a \tau}\left(1-\mathrm{e}^{-a \tau}\right) \\
& =A \mathrm{e}^{-a \tau}+B \mathrm{e}^{-2 a \tau}+C+D \mathrm{e}^{-\lambda \tau}+E \tau \mathrm{e}^{-\lambda \tau} .
\end{aligned}
$$

This is true setting

$$
A=-\alpha \beta_{1}+\frac{\sigma^{2}}{a}, B=-2 a \beta_{2}-\frac{\sigma^{2}}{a}, C=0, D=-\beta_{4} \lambda+\beta_{5}, E=-\beta_{5 \lambda} .
$$

We next prove that $\sigma \mathrm{e}^{-a(\cdot)} \in \operatorname{Im}\left[G_{\beta}(\cdot, \beta)\right]$, or equivalently, we look for real numbers such that

$$
\sigma \mathrm{e}^{-a \tau}=A \mathrm{e}^{-a \tau}+B \mathrm{e}^{-2 a \tau}+C+D \mathrm{e}^{-\lambda \tau}+E \tau \mathrm{e}^{-\lambda \tau}
$$

Setting $A=\sigma$ and $B=C=D=E=0$ the identity follows.

As a conclusion, we have the following corollary:

Corollary 2. For each $\lambda>0$, the Hull and White short rate model is consistent with the forward curve manifold $\mathcal{G}^{\lambda}$ given in (33).

Remark. As in the case of the HL-model, we see that if the initial forward curve is a Nelson-Siegel curve, then the following forward curves are exponentially extended Nelson-Siegel curves, belonging to a particular manifold $\mathcal{G}^{\lambda}$. It must be noted that, even when the forward curve process moves on a wider manifold $\mathcal{H}$ with parameter set $\mathcal{Z}=\mathbb{R}^{6}$,

$$
H(\tau ; \beta)=\beta_{1} \mathrm{e}^{-a \tau}+\beta_{2} \mathrm{e}^{-2 a \tau}+\beta_{3}+\beta_{4} \mathrm{e}^{-\beta_{6} \tau}+\beta_{5} \tau \mathrm{e}^{-\beta_{6} \tau}, \tau \geq 0,
$$

it is not true that this particular manifold is consistent with the HW model. The parameter $\beta_{6}$ must be equal to the parameter $\lambda$ in (1) to get the drift consistency condition (12).

\section{Application to Argentina's Interest Rate}

In this section we apply the Hull-White model to the Argentinian Central Bank bills, named Lebac (Letras del Banco Central). There are seven maturities for the Lebac bills: 35, 63, 98, 120, 147, 203 and 252 days. We have taken the 35d-maturity Lebacs as the short interest rate and we roughly estimated the forward rate curve using the other six maturities:

$$
f\left(0, t_{i}\right)\left(t_{i+1}-t_{i}\right)=\left(\frac{\operatorname{Lebac}\left(0, t_{i+1}\right)}{\operatorname{Lebac}\left(0, t_{i}\right)}-1\right),
$$

where $\operatorname{Lebac}\left(0, t_{i}\right)$ is the price of the Lebac bill with maturity $t_{i}$ at the initial date, $t_{1}=35 d, t_{2}=63 d$, and so on. The data used to calibrate the model parameters are weekly data, from January 6 to December 27, 2016 In order to model the forward rate curves, we set January 4, 2017 as the initial date and calibrated the initial curve to a Nelson and Siegel rate curve. The observed forward 
rates $f\left(0, t_{i}\right)$ for each of the seven maturities are plotted in "Figure 1", together with a Nelson and Siegel curve with $z_{1}=0.19508, z_{2}=0.040724$ and $z_{3}=0.0061793$.

The scale parameter $\lambda$ was estimated from the data: $\lambda=0.051237$ which corresponds to a maximum at thirty five days, or $\lambda=0.3587$ respectively at five weeks.

In "Figure 2" we show the Hull-White forward curves consistent with Nelson and Siegel manifold for specifics days and the corresponding Lebac forward curve observed in the market in the same day, so as to do a back-testing of the models.

The Hull-White model presents a good fit when it is used to make projections in this market for few weeks. The estimated forward curve at the closest date to the initial one presents a good picture comparing it with what actually happened. It follows the same decreasing shape and with less than one basic point of difference, and it continues providing an accurate projection along the time. As $t$ grows, the estimated curve becomes a flat curve, due to the exponential decreasing terms in $t$ that appears in the coefficients of the forward curve formula (31).

Another interesting issue is to test the projection of the Lebac bills in each maturity date. "Figure 3" shows the cross-section of the forward curve for each maturity and the estimated one. Showing the projected evolution for Lebacs at different maturities and the corresponding market data. As in the further case, the decreasing exponential term in $t$ produces a very small estimated volatility and the estimation is acceptable only for some few weeks.

\section{Conclusions}

In the previous sections we presented two families of parametric curves that are consistent with the Ho-Lee and the Hull and White short rate models, respectively. These families contain curves that are extensions of the Nelson and Siegel

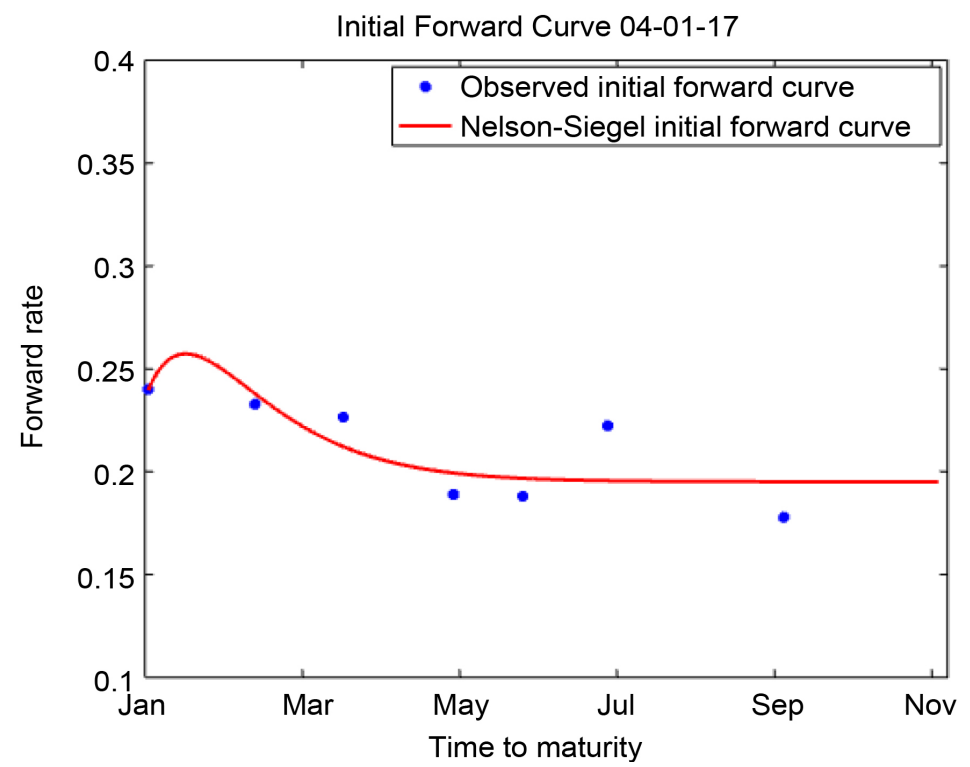

Figure 1. Initial forward rate curve. 

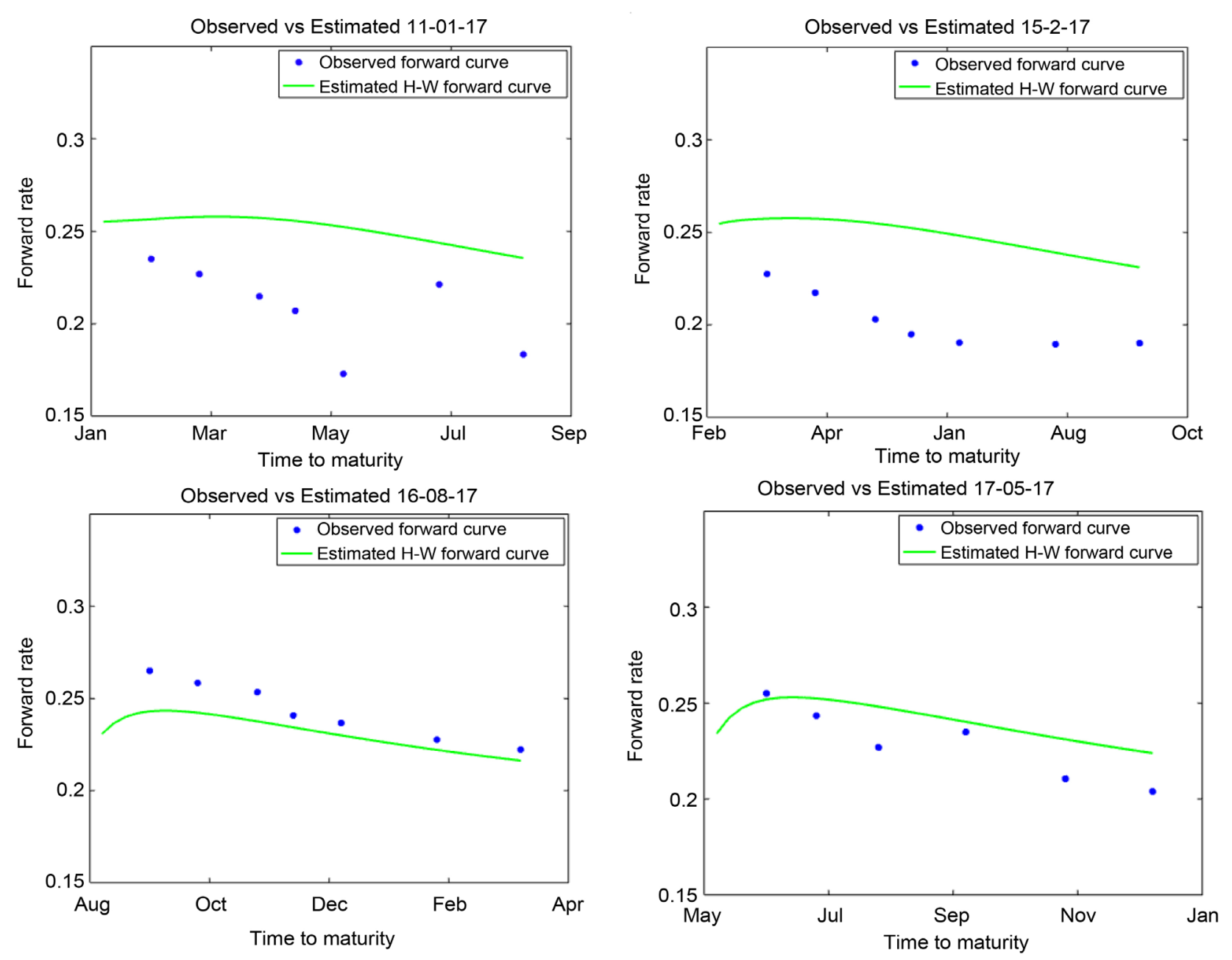

Figure 2. Forward rate curve evolution.

classical curves, in the sense that each element can be written as the sum of a Nelson and Siegel curve plus a linear function in $\tau$ or plus an exponential function in $\tau$. We proved that each of these short rate models is consistent with a such family of forward curve manifolds $\mathcal{G}^{\lambda}$. Moreover, for each $\lambda, G^{\lambda}$ is a set of forward curves driver by four factors. Three of them are the Nelson and Siegel factors $1, \mathrm{e}^{-\lambda \tau}$ and $\tau \mathrm{e}^{-\lambda \tau}$. In the HL model, the fourth factor is a linear function in $\tau$ multiplied by $\sigma$, the short rate volatility. In the HW model it is an exponential decaying function $c_{1} \mathrm{e}^{-a \tau}+c_{2} \mathrm{e}^{-2 a \tau}$, where $a$ is the model parameter. In both cases, the scale parameter $\lambda$ remains the same along the forward rate process.

Nevertheless, it is worth noting that the dynamics of the Nelson and Siegel curve under the hypothesis of an HJM framework does not seem to be a good estimator for the evolution of the observed forward rate curve. In fact, the decreasing exponential terms in the case of the HW-model produce flat curves as $t$ grows, and the linear term $t \tau$ in the HL-model produces too big values that does not represent rate values. A clearly and known reason for this poor estimation is that they are one factor short rate models and so they cannot capture the 

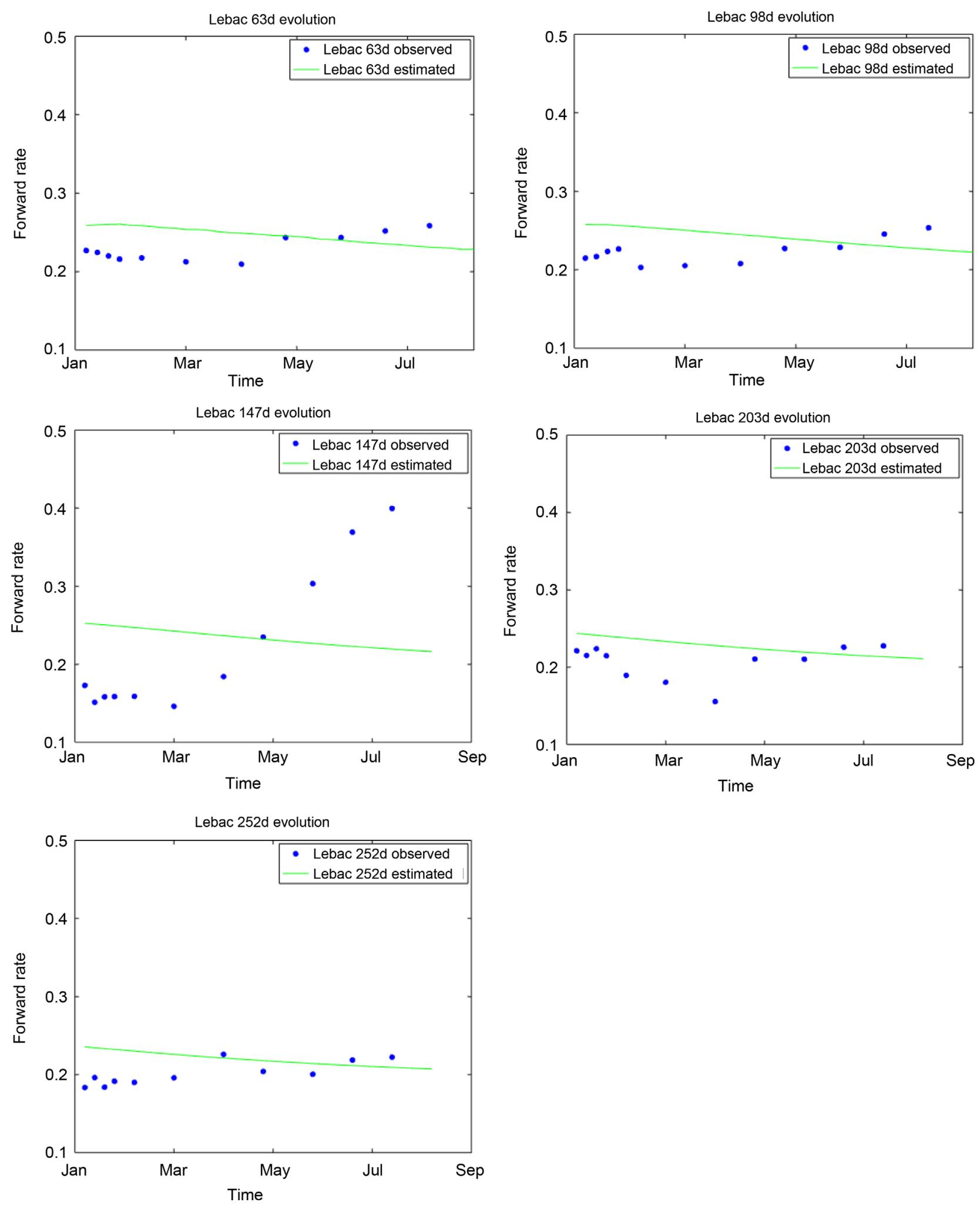

Figure 3. Cross-section of the forward curve.

hole forward curve evolution.

A further research work is to study the consistency of multi-factor short rate 
models with families of Nelson and Siegel curves, so as to incorporate other volatilities term structures and make the forward rate curve evolution more realistic.

\section{References}

[1] Nelson, C. and Siegel, A. (1998) Parsimonious Modeling of Yield Curves. The Journal of Business, 60, 473-489. http://www.jstor.org/stable/2352957

[2] Annaert J., Claes, A.G.P., de Ceuster, M.J.K. and Zhang, H. (2013) Estimating the Yield Curve Using the Nelson-Siegel Model: A Ridge Regression Approach. International Review of Economics and Finance, 27, 482-496. https://doi.org/10.2139/ssrn.2054689

[3] Björk, T. and Christensen, B.J. (1999) Interest Rate Dynamics and Consistent Forward Rate Curves. Mathematical Finance, 9, 323-348. https://doi.org/10.1111/1467-9965.00072

[4] Øksendal, B. (1998) Stochastic Differential Equations. Springer Verlag, Berlin. https://doi.org/10.1007/978-3-662-03620-4

[5] Heath, D., Jarrow, R. and Morton, A. (1992) Bond Pricing and the Term Structure of Interest Rates. Econometrica, 60, 77-106.

https://econpapers.repec.org/article/ecmemetrp/v_3a60_3ay_3a1992_3ai_3a1_3ap_ 3a77-105.htm https://doi.org/10.2307/2951677

[6] Brace, A. and Musiela, M. (1994) A Multifactor Gauss Markov Implementation of Heath, Jarrow, and Morton. Mathematical Finance, 4, 259-283.

https://doi.org/10.1111/j.1467-9965.1994.tb00095.x

[7] Ho, T.S.Y. and Lee, S.B. (1986) Term Structure Movements and Pricing Interest Rate Contingent Claims. The Journal of Finance, 41, 1011-1029. https://doi.org/10.1111/j.1540-6261.1986.tb02528.x

[8] Brigo, D. and Mercurio, F. (2006) Interest Rate Models-Theory and Practice. Springer Verlag, Berlin.

[9] Hull, J. and White, A. (1990) Pricing Interest-Rate-Derivative Securities. The Review of Financial Studies, 3, 573-592. http://www.jstor.org/stable/2962116 https://doi.org/10.1093/rfs/3.4.573 